\title{
Universal coherence protection in a solid-state spin qubit
}

\author{
Kevin C. Miao', Joseph P. Blanton ${ }^{1,2}$, Christopher P. Anderson ${ }^{1,2}$, Alexandre Bourassa ${ }^{1}$, Alexander L. Crook ${ }^{1,2}$, \\ Gary Wolfowicz $^{1,4}$, Hiroshi Abe ${ }^{3}$, Takeshi Ohshima ${ }^{3}$, David D. Awschalom ${ }^{1,2,4 *}$ \\ 1Pritzker School of Molecular Engineering, University of Chicago, Chicago, IL 60637, USA. ²Department of Physics, University of Chicago, Chicago, IL 60637, USA. ${ }^{3}$ National \\ Institutes for Quantum and Radiological Science and Technology, 1233 Watanuki, Takasaki, Gunma 370-1292, Japan. ${ }^{4}$ Center for Molecular Engineering and Materials \\ Science Division, Argonne National Laboratory, Lemont, IL 60439, USA. \\ ${ }^{*}$ Corresponding author. E-mail: awsch@uchicago.edu
}

Decoherence limits the physical realization of qubits and its mitigation is critical for the development of quantum science and technology. We construct a robust qubit embedded in a decoherence-protected subspace, obtained by applying microwave dressing to a clock transition of the ground-state electron spin of a silicon carbide divacancy defect. The qubit is universally protected from magnetic, electric, and temperature fluctuations, which account for nearly all relevant decoherence channels in the solid state. This culminates in an increase of the qubit's inhomogeneous dephasing time by over four orders of magnitude (to $>22$ milliseconds), while its Hahn-echo coherence time approaches 64 milliseconds. Requiring few key platform-independent components, this result suggests that substantial coherence improvements can be achieved in a wide selection of quantum architectures.

Electron spins embedded in a solid-state host, such as silicon carbide ( $\mathrm{SiC}$ ) and diamond, are attractive platforms for quantum information processing by virtue of their optical interface $(1,2)$ and engineered interactions with the host crystal (3-6).

These electron spins are highly controllable using external magnetic fields because of the electron's large magnetic moment. However, unwanted magnetic field noise will also couple intensely through this degree of freedom and quench spin coherences $(7,8)$. In particular, a naturally abundant host crystal typically has nonzero nuclear spin isotopes and paramagnetic impurities, whose fluctuations produce variations in the magnetic field at the electron spin with magnitudes as large as $0.45 \mathrm{mT}(9)$.

These magnetic field fluctuations limit the electron spin's Hahn-echo coherence time $\left(T_{2}\right)$ to around a millisecond $(6,7$, $10,11)$ and suppress spin inhomogeneous dephasing times $\left(T_{2}^{*}\right)$ to a few microseconds $(10,11)$. These relatively short inhomogeneous dephasing times typically bound the timescales for quantum information storage and manipulation, requiring the development of nuclear spin quantum memories $(12,13)$, while also limiting the fidelity of quantum information transfer to coupled systems, such as superconducting resonators (14). These restrictions call for the extension of electron spin coherence, which is typically achieved by means of dynamical decoupling (15), isotopic purification $(13,16,17)$, or Hamiltonian engineering $(3,4,18,19)$.

One particular solid-state electron spin system, the basally oriented $k h$ divacancy in the $4 \mathrm{H}$ polytype of $\mathrm{SiC}$, has recently been shown to have reduced sensitivity to magnetic noise when operated near zero external magnetic field (20). This robustness primarily arises from the transverse zero-field splitting (ZFS) intrinsic to the spin system's single mirror plane symmetry (Fig. 1A, lower left). When combined with the spin system's axial ZFS, clock transitions emerge near $\mathbf{B}=0$. By operating at this point, the spin has lengthened inhomogeneous dephasing times, among other properties (21). We implement a protocol to further extend the ground-state spin inhomogeneous dephasing time, as well as the Hahn-echo coherence time, of a single $k h$ divacancy in $4 \mathrm{H}-\mathrm{SiC}$ by simply applying a continuous microwave dressing drive resonant with two of the three ground-state spin-1 sublevels at zero field. The combination of a clock transition and periodic driving allows for the advantages of both methods to manifest, while the individual weaknesses are diminished (21). Furthermore, there are many practical advantages of this protocol when compared to typical pulsed dynamical decoupling sequences, including uninterrupted coherence protection, lower peak drive powers, and fewer non-idealities in implementing the protocol. Importantly, this dressed spin system retains its large magnetic and electric response to resonant microwave control fields, preserving the capability to rapidly perform quantum operations to coherently manipulate the long-lived quantum state and the ability to prepare arbitrary superpositions in this new basis.

We isolate the $k h$ divacancy used in this study in naturally abundant, commercially available $4 \mathrm{H}-\mathrm{SiC}$ at $5 \mathrm{~K}(21)$. The ground-state spin-1 system is initialized and read out through optical pulses, while spin rotations are produced by microwave-frequency magnetic and electric fields from on-chip wires and capacitors, respectively (Fig. 1A). A three-axis electromagnet provides vector control of the external magnetic field along the Cartesian axes of the spin system (Fig. 1A, 
lower left). At $\mathbf{B}=0$, a spin transition can be driven between the upper two spin states, $\quad|+\rangle=\frac{1}{\sqrt{2}}\left(\left|+1_{z}\right\rangle+\left|-1_{z}\right\rangle\right) \quad$ and $|-\rangle=\frac{1}{\sqrt{2}}\left(\left|+1_{z}\right\rangle-\left|-1_{z}\right\rangle\right)$ (where $\left| \pm 1_{z}\right\rangle$ denote the $m_{s}= \pm 1$ sublevels in the $S_{z}$ basis). Ramsey interferometry of the $|+\rangle \leftrightarrow|-\rangle$ transition is used to determine the transverse ZFS magnitude $E /(2 \pi)=18.353164(4) \mathrm{MHz}$ to a high degree of precision and accuracy (21), allowing us to apply a continuous microwave-frequency magnetic drive at an angular frequency $\omega=2 E$ resonant with the $|+\rangle \leftrightarrow|-\rangle$ transition. At a sufficiently high drive Rabi frequency $\Omega$, the $| \pm\rangle$ spin levels undergo Autler-Townes splitting (21) to form dressed spin states $| \pm 1\rangle$ with energy levels offset from that of $|+\rangle$ by $\pm \Omega / 2$. These dressed states form the basis for the decoherenceprotected subspace (DPS), and can be observed by applying a weaker microwave probe pulse and sweeping its frequency detuning $\Delta$ from the $|0\rangle \leftrightarrow|+\rangle$ transition frequency (Fig. 1C). For subsequent measurements, we operate at $\Omega /(2 \pi)=350 \mathrm{kHz}$ to mitigate higher-order energy dispersion components (21). We realize coherent control within the dressed spin-1 system by driving $\Delta m_{\mathrm{s}}= \pm 1$ transitions $|0\rangle \leftrightarrow| \pm 1\rangle$ with ac magnetic fields (Fig. 1D) and a $\Delta m_{\mathrm{s}}$ $= \pm 2$ transition $|+1\rangle \leftrightarrow|-1\rangle$ with ac electric fields (22) (Fig. 1E). Readout of the $| \pm 1\rangle$ basis is accomplished by non-adiabatically disabling the dressing drive, rotating into the $\{|0\rangle,|+\rangle\}$ basis, and optically probing the spin population in $|0\rangle$ (21).

The energy inhomogeneity of the driven spin system can be quantified by preparing a superposition $\left|\psi_{0}\right\rangle=\frac{1}{\sqrt{2}}(|+1\rangle+|-1\rangle)$ in the DPS and executing a Ramsey free precession sequence. With active feedback procedures in place, we measure a $T_{2}^{*}$ spin inhomogeneous dephasing time in this basis to be 22.4(10) milliseconds (Fig. 2A), which is over four and two orders of magnitude longer than the $T_{2}^{*}$ measured for the same $k h$ divacancy at $B_{z}=1.2 \mathrm{mT}$ and $\mathbf{B}=0$, respectively (Fig. 2B). Moreover, by adding a single refocusing pulse, we extend the lifetime of the superposition to a $T_{2}$ Hahn-echo spin coherence time of 64(4) milliseconds (Fig. 2C). The mechanism by which the coherence is protected in the DPS can be understood by examining the Hamiltonian with a dressing drive applied on resonance, in the frame rotating at the drive frequency, $\omega=2 E$,

$$
\tilde{H} / \hbar=\left[\begin{array}{ccc}
0 & \gamma_{e} B_{x} e^{-i E t} & \frac{\Omega}{2}+\gamma_{e} B_{z} e^{-2 i E t} \\
\gamma_{e} B_{x} e^{i E t} & -D & -i \gamma_{e} B_{y} e^{i E t} \\
\frac{\Omega}{2}+\gamma_{e} B_{z} e^{2 i E t} & i \gamma_{e} B_{y} e^{-i E t} & 0
\end{array}\right],
$$

where $\gamma_{e}$ is the electron gyromagnetic ratio, $D$ is the magnitude of the axial ZFS, and $\Omega$ is the dressing drive Rabi frequency. As a first-order approximation, the magnetic noise contributions perturb the spin only when the matrix element becomes time-independent. For $B_{x}$ and $B_{y}$ noise contributions, this is achieved for frequency components at $\pm E$. For $B_{z}$ contributions, this is achieved for frequency components at $\pm 2 E$. Thus, within the DPS, an effective bandpass filter is applied on the magnetic noise, with passbands located at $\pm E$ and $\pm 2 E$ each with bandwidths proportional to $\Omega$. Combined with the $1 / f$ dependence of noise power spectra, this results in a greatly reduced sensitivity to magnetic noise.

We then examine the energy dispersion relations between the dressed spin levels under magnetic, electric, and temperature fluctuations in order to reveal their contributions to inhomogeneity in the DPS. We first consider the effect of magnetic fields on the dressed spin levels, as magnetic noise typically limits the coherence of many electron spin systems. Under a continuous drive resonant with the $|+\rangle \leftrightarrow|-\rangle$ transition, the dispersion relation of the ground-state spin acquires both quadratic and quartic dependences on magnetic field (21). The dispersion relation is probed by applying magnetic fields along the $x$ - and $z$-axes of the spin system while scanning the frequency detuning $\Delta$ of a probe drive (Fig. $3 \mathrm{~A}, \mathrm{~B})$. Rotational symmetry of the system about the $z$-axis leads to indistinguishable effects from $x$ - and $y$-axis fields (21). The resulting frequencies of the $|0\rangle \leftrightarrow| \pm 1\rangle$ spin resonances provide us with the driven spin system's spectral response to magnetic fields. To confirm these observations, we develop an analytical model of the driven spin system using Floquet analysis alongside a numerical model through spin-1 master equation simulations (21). We find excellent agreement between the experimental data and these models (Fig. $3 \mathrm{C}, \mathrm{D})$. We then consider the primary source of magnetic noise, the nuclear spin bath, as an isotropic magnetic fluctuator with an estimated fluctuation magnitude at this $k h$ divacancy to be $13 \mu \mathrm{T}$ (21). We perform fine scans of the $|+1\rangle \leftrightarrow|-1\rangle$ transition using Ramsey interferometry (Fig. $3 \mathrm{E}, \mathrm{F})$ and confirm the high degree of insensitivity in this range, illustrating the primary mechanism for the largely increased spin coherence times. These results, once combined with a phenomenological model of the spin energy inhomogeneity (21), allow us to quantitatively confirm that residual inhomogeneity from magnetic fields still plays a role in limiting the spin coherence, even after substantial suppression in the DPS.

We can then apply the analytical energy dispersion relations to understand the effect of electric noise and temperature fluctuations on spin inhomogeneous dephasing in the DPS. Electric and temperature fluctuations affect the axial and transverse ZFS magnitudes, as well as a corresponding 
detuning of the dressing drive from the resonance frequency $\omega=2 E$. We use the undriven spin's first-order sensitivity to electric fields (23) at $\mathbf{B}=0$ to quantify the magnitude of electric field noise present in the system (21). In the DPS, we gain first-order protection against fluctuations in the ZFS magnitudes, resulting in increased robustness against electric field noise and temperature shifts. This leads to a reduction of electrically induced spin energy inhomogeneity by nearly two orders of magnitude (21), and diminishes electric field contributions in limiting the spin coherence in the DPS. Further reduction of electric field contributions may emerge by applying a dc electric field to deplete fluctuating charges $(6,20)$, potentially leading to nearly complete elimination of electric field noise.

The energy dispersion relations in the driven basis confirm that the spin's energy levels depend linearly on the dressing drive Rabi frequency. Hence, amplitude drifts of the dressing drive and corresponding fluctuations in the Rabi frequency $\delta \Omega$ introduce first-order inhomogeneity of the dressed spin levels and cause shortening of the measured $T_{2}^{*}$. To this end, we implement active feedback of the dressed spin resonance frequency in order to counteract these slow drifts and reduce inhomogeneity (Fig. 4A). By evaluating an error signal derived from Ramsey free precession of the dressed spin (Fig. 4B), we measure and correct drifts on the order of $30 \mathrm{~Hz}$ in the spin resonance frequency (21), consistent with the 100 $\mathrm{ppm} /{ }^{\circ} \mathrm{C}$ stability of the dressing drive oscillator. In order to validate the effectiveness of our feedback protocol, we measure Ramsey free precession of $\left|\psi_{0}\right\rangle=\frac{1}{\sqrt{2}}(|+1\rangle+|-1\rangle)$ with and without feedback enabled (Fig. 4C). With active feedback enabled, we measure our reported $T_{2}^{*}$ of 22.4(10) ms; when disabled, this value shortens to $17.4(10) \mathrm{ms}$ as the dressing drive inhomogeneity suppresses the spin's coherence on the timescale of hours (Fig. 4D). These results suggest that by incorporating this feedback protocol, we have largely mitigated the effects of dressing drive amplitude fluctuations and that noise sources intrinsic to the host crystal remain as the limiting factor for spin coherence in the DPS.

The few requirements for successful implementation of this protocol allow for immediate extension to other quantum systems. Specifically, a candidate system must have a coherently controllable clock transition, where upon being driven the resulting dressed states are also coherently controllable (21). Other basally oriented defects in SiC (24-26) have transverse ZFS magnitudes larger than what has been observed here, and are expected to experience even greater coherence protection. Strained diamond nitrogen-vacancy centers (14) may also stand to benefit from this protocol. Turning to other systems, donor spins in silicon with robust clock transitions $(27,28)$, superconducting qubits operated at a degeneracy point (29), and molecular spins with ZFS or hyperfine interactions $(30,31)$ may be driven to produce a DPS.

With an extended spin $T_{2}^{*}$, the prospects for unity-cooperativity systems between single solid-state spins and weakly interacting quantum systems are more promising. Hartmann-Hahn coupling between a pair of spins prepared in the DPS can mediate two-qubit interactions (18). By mitigating strain inhomogeneity through material improvements or dynamical decoupling, an ensemble of $n$ driven spins can attain a $\sqrt{n}$ enhancement in coupling strength, providing a nearterm avenue toward a high-cooperativity coupled system. We have shown that the qubit in the DPS is still highly responsive to resonantly applied magnetic and electric control fields, and thus magnetic, electric, and acoustic $(3,5)$ interactions can be used for coupling. Lastly, megahertz-scale tuning of the dressed spin energy levels allows for rapid adjustment of interaction strengths, enabling the spin to be an efficient quantum bus.

This protocol is compatible with additional forms of noise suppression, including isotopic purification, charge depletion, and pulsed dynamical decoupling. The residual electron spin energy shifts can be decreased significantly by reducing the volume density of fluctuating spins in the bath $(13,16,17)$. Dynamic techniques such as spin bath driving (11) and hyperpolarization (32) may further reduce these contributions. Charge depletion, which has led to near-transform-limited optical linewidths in divacancies $(6,20)$, can further suppress electric field contributions. We have presently demonstrated compatibility with pulsed dynamical decoupling using a Hahn-echo sequence; higher-order dynamical decoupling sequences may allow for spin coherence to rapidly reach the spin-lattice relaxation limit with few control pulses. Considering these prospective avenues, this dressing protocol represents a major development for potentially improving coherence across a variety of quantum systems, and is a crucial step toward integrating $\mathrm{SiC}$ divacancies into robust, realworld quantum technologies.

\section{REFERENCES AND NOTES}

1. L. Robledo, L. Childress, H. Bernien, B. Hensen, P. F. A. Alkemade, R. Hanson, Highfidelity projective read-out of a solid-state spin quantum register. Nature 477 , 574-578 (2011). doi:10.1038/nature10401 Medline

2. D. J. Christle, P. V. Klimov, C. F. de las Casas, K. Szász, V. Ivády, V. Jokubavicius, J. UI Hassan, M. Syväjärvi, W. F. Koehl, T. Ohshima, N. T. Son, E. Janzén, Á. Gali, D. D. Awschalom, Isolated spin qubits in SiC with a high-fidelity infrared spin-tophoton interface. Phys. Rev. X 7, 021046 (2017). doi:10.1103/PhysRevX.7.021046

3. A. Barfuss, J. Teissier, E. Neu, A. Nunnenkamp, P. Maletinsky, Strong mechanical driving of a single electron spin. Nat. Phys. 11, 820-824 (2015). doi:10.1038/nphys3411

4. E. R. MacQuarrie, T. A. Gosavi, S. A. Bhave, G. D. Fuchs, Continuous dynamical decoupling of a single diamond nitrogen-vacancy center spin with a mechanical resonator. Phys. Rev. B Condens. Matter Mater. Phys. 92, 224419 (2015). doi:10.1103/PhysRevB.92.224419

5. S. J. Whiteley, G. Wolfowicz, C. P. Anderson, A. Bourassa, H. Ma, M. Ye, G. Koolstra, K. J. Satzinger, M. V. Holt, F. J. Heremans, A. N. Cleland, D. I. Schuster, G. Galli, D. D. Awschalom, Spin-phonon interactions in silicon carbide addressed by 
Gaussian acoustics. Nat. Phys. 15, 490-495 (2019). doi:10.1038/s41567-0190420-0

6. C. P. Anderson, A. Bourassa, K. C. Miao, G. Wolfowicz, P. J. Mintun, A. L. Crook, H. Abe, J. U. Hassan, N. T. Son, T. Ohshima, D. D. Awschalom, Electrical and optical control of single spins integrated in scalable semiconductor devices. Science 1230, 1225-1230 (2019).

7. H. Seo, A. L. Falk, P. V. Klimov, K. C. Miao, G. Galli, D. D. Awschalom, Quantum decoherence dynamics of divacancy spins in silicon carbide. Nat. Commun. 7 , 12935 (2016). doi:10.1038/ncomms12935 Medline

8. P. L. Stanwix, L. M. Pham, J. R. Maze, D. Le Sage, T. K. Yeung, P. Cappellaro, P. R. Hemmer, A. Yacoby, M. D. Lukin, R. L. Walsworth, Coherence of nitrogen-vacancy electronic spin ensembles in diamond. Phys. Rev. B Condens. Matter Mater. Phys. 82, 201201 (2010). doi:10.1103/PhysRevB.82.201201

9. P. V. Klimov, A. L. Falk, D. J. Christle, V. V. Dobrovitski, D. D. Awschalom, Quantum entanglement at ambient conditions in a macroscopic solid-state spin ensemble. Sci. Adv. 1, e1501015 (2015). doi:10.1126/sciadv.1501015 Medline

10. D. J. Christle, A. L. Falk, P. Andrich, P. V. Klimov, J. U. Hassan, N. T. Son, E. Janzén, T. Ohshima, D. D. Awschalom, Isolated electron spins in silicon carbide with millisecond coherence times. Nat. Mater. 14, 160-163 (2015). doi:10.1038/nmat4144 Medline

11. E. Bauch, C. A. Hart, J. M. Schloss, M. J. Turner, J. F. Barry, P. Kehayias, S. Singh, R. L. Walsworth, Ultralong Dephasing Times in Solid-State Spin Ensembles via Quantum Control. Phys. Rev. X 8, 031025 (2018). doi:10.1103/PhysRevX.8.031025

12. G. D. Fuchs, G. Burkard, P. V. Klimov, D. D. Awschalom, A quantum memory intrinsic to single nitrogen-vacancy centres in diamond. Nat. Phys. 7, 789-793 (2011). doi:10.1038/nphys2026

13. P. C. Maurer, G. Kucsko, C. Latta, L. Jiang, N. Y. Yao, S. D. Bennett, F. Pastawski, D. Hunger, N. Chisholm, M. Markham, D. J. Twitchen, J. I. Cirac, M. D. Lukin, Roomtemperature quantum bit memory exceeding one second. Science 336, 12831286 (2012). doi:10.1126/science.1220513 Medline

14. Y. Kubo, F. R. Ong, P. Bertet, D. Vion, V. Jacques, D. Zheng, A. Dréau, J. F. Roch, A. Auffeves, F. Jelezko, J. Wrachtrup, M. F. Barthe, P. Bergonzo, D. Esteve, Strong coupling of a spin ensemble to a superconducting resonator. Phys. Rev. Lett. 105, 140502 (2010). doi:10.1103/PhysRevLett.105.140502 Medline

15. M. H. Abobeih, J. Cramer, M. A. Bakker, N. Kalb, M. Markham, D. J. Twitchen, T. H. Taminiau, One-second coherence for a single electron spin coupled to a multiqubit nuclear-spin environment. Nat. Commun. 9, 2552 (2018). doi:10.1038/s41467-018-04916-z Medline

16. E. D. Herbschleb, H. Kato, Y. Maruyama, T. Danjo, T. Makino, S. Yamasaki, I. Ohki, K. Hayashi, H. Morishita, M. Fujiwara, N. Mizuochi, Ultra-long coherence times amongst room-temperature solid-state spins. Nat. Commun. 10, 3766 (2019). doi:10.1038/s41467-019-11776-8 Medline

17. A. Bourassa, C. P. Anderson, K. C. Miao, M. Onizhuk, H. Ma, A. L. Crook, H. Abe, J. UI Hassan, T. Ohshima, N. T. Son, G. Galli, D. D. Awschalom, Entanglement and control of single quantum memories in isotopically engineered silicon carbide. arXivi2005.07602 [quant-ph] (2020).

18. A. Laucht, R. Kalra, S. Simmons, J. P. Dehollain, J. T. Muhonen, F. A. Mohiyaddin, S. Freer, F. E. Hudson, K. M. Itoh, D. N. Jamieson, J. C. McCallum, A. S. Dzurak, A. Morello, A dressed spin qubit in silicon. Nat. Nanotechnol. 12, 61-66 (2017). doi:10.1038/nnano.2016.178 Medline

19. J. Teissier, A. Barfuss, P. Maletinsky, Hybrid continuous dynamical decoupling: A photon-phonon doubly dressed spin. J. Opt.19, 044003 (2017). doi:10.1088/2040-8986/aa5f62

20. K. C. Miao, A. Bourassa, C. P. Anderson, S. J. Whiteley, A. L. Crook, S. L. Bayliss, G. Wolfowicz, G. Thiering, P. Udvarhelyi, V. Ivády, H. Abe, T. Ohshima, Á. Gali, D. D. Awschalom, Electrically driven optical interferometry with spins in silicon carbide. Sci. Adv. 5, eaay0527 (2019). doi:10.1126/sciadv.aay0527 Medline

21. See Supplementary Materials.

22. P. V. Klimov, A. L. Falk, B. B. Buckley, D. D. Awschalom, Electrically driven spin resonance in silicon carbide color centers. Phys. Rev. Lett. 112, 087601 (2014), doi:10.1103/PhysRevLett.112.087601

23. P. Jamonneau, M. Lesik, J. P. Tetienne, I. Alvizu, L. Mayer, A. Dréau, S. Kosen, J. F. Roch, S. Pezzagna, J. Meijer, T. Teraji, Y. Kubo, P. Bertet, J. R. Maze, V. Jacques, Competition between electric field and magnetic field noise in the decoherence of a single spin in diamond. Phys. Rev. B 93, 024305 (2016) doi:10.1103/PhysRevB.93.024305

24. A. L. Falk, B. B. Buckley, G. Calusine, W. F. Koehl, V. V. Dobrovitski, A. Politi, C. A. Zorman, P. X. L. Feng, D. D. Awschalom, Polytype control of spin qubits in silicon carbide. Nat. Commun. 4, 1819 (2013). doi:10.1038/ncomms2854 Medline

25. Z. Mu, S. A. Zargaleh, H. J. von Bardeleben, J. E. Fröch, H. Cai, X. Yang, J. Yang, X. Li, I. Aharonovich, W. Gao, Coherent manipulation with resonant excitation and single emitter creation of nitrogen vacancy centers in $4 \mathrm{H}$ silicon carbide. Nano Lett. (2020). doi:10.1021/acs.nanolett.0c02342 Medline

26. K. Khazen, H. J. Von Bardeleben, S. A. Zargaleh, J. L. Cantin, M. Zhao, W. Gao, T. Biktagirov, U. Gerstmann, High-resolution resonant excitation of NV centers in 6H-SiC: A matrix for quantum technology applications. Phys. Rev. B 100, 205202 (2019). doi:10.1103/PhysRevB.100.205202

27. G. Wolfowicz, A. M. Tyryshkin, R. E. George, H. Riemann, N. V. Abrosimov, P. Becker, H. J. Pohl, M. L. W. Thewalt, S. A. Lyon, J. J. L. Morton, Atomic clock transitions in silicon-based spin qubits. Nat. Nanotechnol. 8, 561-564 (2013). doi:10.1038/nnano.2013.117 Medline

28. K. J. Morse, P. Dluhy, J. Huber, J. Z. Salvail, K. Saeedi, H. Riemann, N. V. Abrosimov, P. Becker, H. J. Pohl, S. Simmons, M. L. W. Thewalt, Zero-field optical magnetic resonance study of phosphorus donors in 28-silicon. Phys. Rev. B 97, 115205 (2018). doi:10.1103/PhysRevB.97.115205

29. M. H. Devoret, A. Wallraff, J. M. Martinis, Superconducting Qubits: A Short Review. arXiv:cond-mat/0411174 [cond-mat.mes-hall] (2004)..

30. S. L. Bayliss, D. W. Laorenza, P. J. Mintun, B. Diler, D. E. Freedman, D. D. Awschalom, Optically addressable molecular spins for quantum information processing. arXiv:2004.07998 [quant-ph] (2020).

31. M. Shiddiq, D. Komijani, Y. Duan, A. Gaita-Ariño, E. Coronado, S. Hill, Enhancing coherence in molecular spin qubits via atomic clock transitions. Nature 531, 348351 (2016). doi:10.1038/nature16984 Medline

32. I. Schwartz, J. Scheuer, B. Tratzmiller, S. Müller, Q. Chen, I. Dhand, Z. Y. Wang, C. Müller, B. Naydenov, F. Jelezko, M. B. Plenio, Robust optical polarization of nuclear spin baths using Hamiltonian engineering of nitrogen-vacancy center quantum dynamics. Sci. Adv. 4, eaat8978 (2018). doi:10.1126/sciadv.aat8978 Medline

33. K. C. Miao, J. P. Blanton, C. P. Anderson, A. Bourassa, A. L. Crook, G. Wolfowicz, H. Abe, T. Ohshima, D. D. Awschalom, Datasets for "Universal coherence protection in a solid-state spin qubit", Version 1.0, Zenodo (2020); https:doi.org/10.5281/zenodo.3975269

34. S. K. Son, S. Han, S. I. Chu, Floquet formulation for the investigation of multiphoton quantum interference in a superconducting qubit driven by a strong ac field. Phys. Rev. A 79, 032301 (2009). doi:10.1103/PhysRevA.79.032301

35. M. S. Rudner, N. H. Lindner, Floquet topological insulators: from band structure engineering to novel non-equilibrium quantum phenomena. arXiv:1909.02008 [cond-mat.mes-hall] (2019).

36. K. I. Seetharam, thesis, California Institute of Technology (2018).

37. X. Xu, Z. Wang, C. Duan, P. Huang, P. Wang, Y. Wang, N. Xu, X. Kong, F. Shi, X. Rong, J. Du, Coherence-protected quantum gate by continuous dynamical decoupling in diamond. Phys. Rev. Lett. 109, 070502 (2012). doi:10.1103/PhysRevLett.109.070502 Medline

38. A. Messiah, Quantum Mechanics (North-Holland, 1962), vol. 2.

39. A. L. Falk, P. V. Klimov, B. B. Buckley, V. Ivády, I. A. Abrikosov, G. Calusine, W. F. Koehl, A. Gali, D. D. Awschalom, Electrically and mechanically tunable electron spins in silicon carbide color centers. Phys. Rev. Lett. 112, 187601 (2014). doi:10.1103/PhysRevLett.112.187601 Medline

40. M. W. Doherty, V. M. Acosta, A. Jarmola, M. S. J. Barson, N. B. Manson, D. Budker, L. C. L. Hollenberg, Temperature shifts of the resonances of the NV-center in diamond. Phys. Rev. B Condens. Matter Mater. Phys. 90, 041201 (2014). doi:10.1103/PhysRevB.90.041201

41. Y. Zhou, J. Wang, X. Zhang, K. Li, J. Cai, W. Gao, Self-Protected Thermometry with Infrared Photons and Defect Spins in Silicon Carbide. Phys. Rev. Appl. 8, 044015 (2017). doi:10.1103/PhysRevApplied.8.044015 


\section{ACKNOWLEDGMENTS}

We thank M. Fukami, S. L. Bayliss, Y. Tsaturyan, P. C. Jerger, V. V. Dobrovitski, M. Onizhuk, and A. A. Clerk for fruitful discussions and comments, and for careful reading of the manuscript. Funding: K.C.M, J.P.B., C.P.A, A.B., A.L.C, G.W., and D.D.A. were supported by DARPA D18AC00015KK1932, AFOSR FA9550-19-10358, ONR N00014-17-1-3026, and the UChicago MRSEC NSF DMR-1420709. H.A. and T.O. were supported by JSPS KAKENHI $18 \mathrm{HO} 3770$ and $20 \mathrm{H} 00355$. This work made use of the Pritzker Nanofabrication Facility part of the Pritzker School of Molecular Engineering at the University of Chicago, which receives support from Soft and Hybrid Nanotechnology Experimental (SHyNE) Resource (NSF ECCS-1542205), a node of the National Science Foundation's National Nanotechnology Coordinated Infrastructure. Author contributions: K.C.M. conceived and designed the experiment, and carried out theoretical calculations. K.C.M. and J.P.B. performed the experiments, with the assistance of G.W. C.P.A. annealed and fabricated the sample. K.C.M., J.P.B., A.B., and A.L.C. developed the confocal microscope setup. H.A. and T.O. performed electron irradiation of the SiC samples. D.D.A. advised on all fronts. All authors contributed to manuscript revision and preparation. Competing interests: K.C.M. and D.D.A. are inventors on a provisional patent application that has been filed relating to this work. All other authors declare that they have no competing interests. Data and materials availability: The data within this study are available on Zenodo (33).

\section{SUPPLEMENTARY MATERIALS}

science.sciencemag.org/cgi/content/full/science.abc5186/DC1

Materials and Methods

Figs. S1 to S3

Supplementary Text

References (34-41)

28 April 2020; accepted 31 July 2020

Published online 13 August 2020

10.1126/science.abc5186 

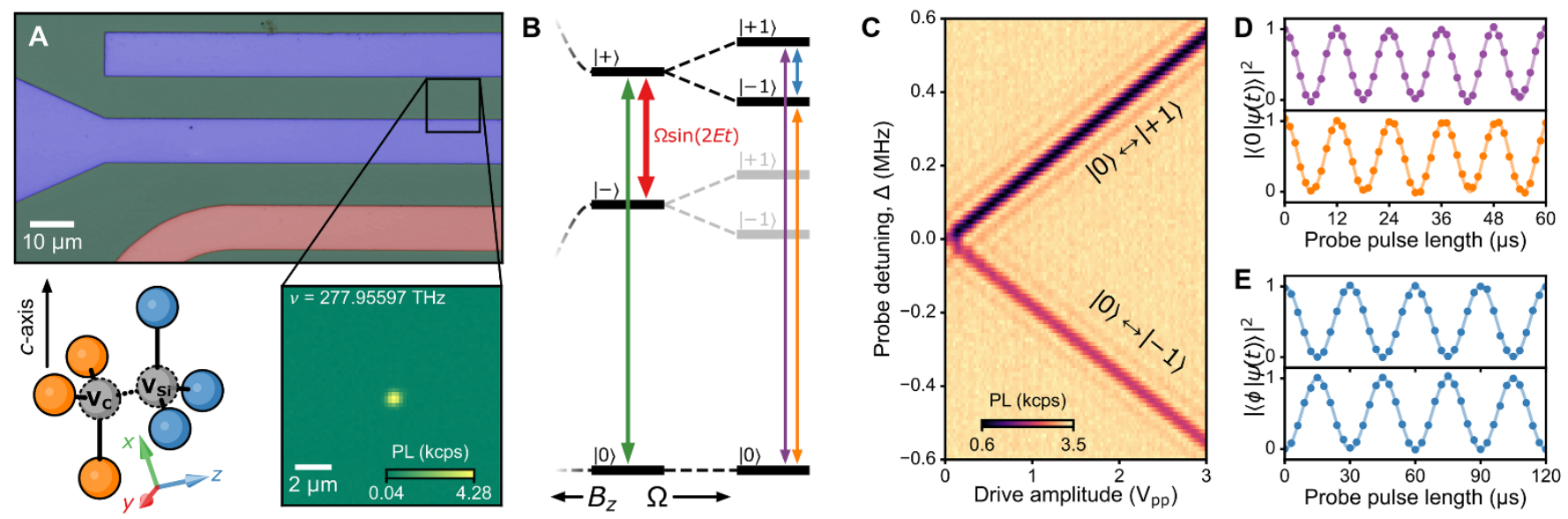

Fig. 1. Driven $k h$ divacancy spin system in $4 \mathrm{H}-\mathrm{SiC}$. (A) False-color optical microscope image of the $4 \mathrm{H}-\mathrm{SiC}$ sample showing an on-chip capacitor (blue) and wire (red) for microwave manipulation of the spin. Inset: Single $k h$ divacancy under resonant optical excitation. Lower left: Lattice diagram of a $k h$ divacancy and nearestneighbor carbon atoms (blue) and silicon atoms (orange). Cartesian axes of the $k h$ divacancy shown as colored vectors. (B) Energy diagram of the $k h$ divacancy ground-state spin in the ${ }^{3} A_{2}$ orbital. At $B=0$, clock transitions form between the three spin-1 levels. Spin manipulation in this basis consists of driving $|0\rangle \leftrightarrow|+\rangle$ (green) and $|+\rangle \leftrightarrow|-\rangle$ (red). Sufficiently strong driving of $|+\rangle \leftrightarrow|-\rangle$ induces Autler-Townes splitting, forming a hybridized spin-photon system, with an accessible upper branch and an inaccessible lower branch (gray). In this basis, spin manipulation consists of magnetically driving $|0\rangle \leftrightarrow| \pm 1\rangle$ (purple, orange), or electrically driving $|+1\rangle \leftrightarrow|-1\rangle$ (blue). (C) Pulsed optically detected magnetic resonance resolving Autler-Townes splitting of the ground-state spin. $\Delta$ is the probe frequency detuning from the $|0\rangle \leftrightarrow|+\rangle$ resonance frequency. (D) Magnetically driven Rabi oscillations using the $|0\rangle \leftrightarrow|+1\rangle$ (upper) and $|0\rangle \leftrightarrow|-1\rangle$ (lower) transitions corresponding to the purple and orange arrows in (B). (E) Electrically driven Rabi oscillations using the transition $|+1\rangle \leftrightarrow|-1\rangle$ highlighted by the blue arrow in (B) after reading out the population of $|+1\rangle(\varphi=+1)$ (upper) and $|-1\rangle(\varphi=-1)$ (lower). 


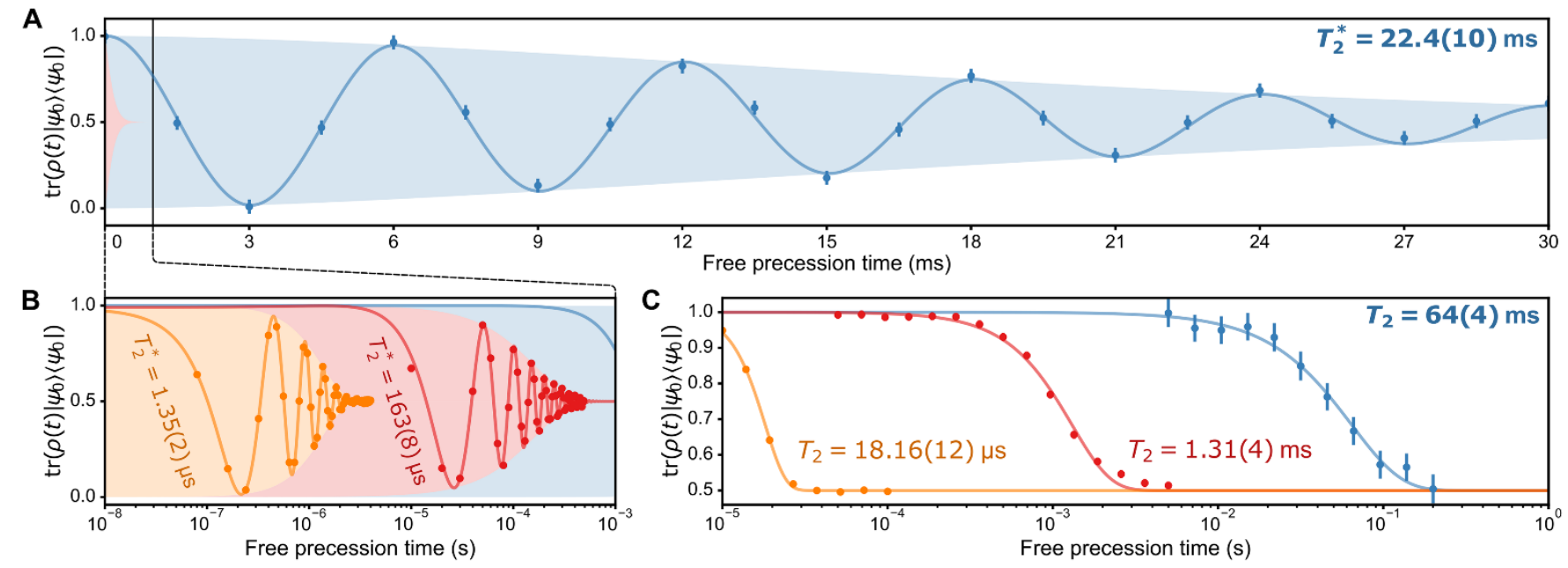

Fig. 2. Spin coherence in the DPS. (A) Ramsey free precession of $\left|\psi_{0}\right\rangle=\frac{1}{\sqrt{2}}(|+1\rangle+|-1\rangle)$ in the DPS at B $=0 \mathrm{mT}$ (blue). A microwave detuning of $+166.6 \mathrm{~Hz}$ is added to increase visibility of the decay envelope. (B) Ramsey free precession of $\left|\psi_{0}\right\rangle=\frac{1}{\sqrt{2}}(|0\rangle+|+\rangle)$ (red) and $\left|\psi_{0}\right\rangle=\frac{1}{\sqrt{2}}\left(|0\rangle+\left|+1_{z}\right\rangle\right)$ (orange) prepared outside of the DPS at B $=0$ $\mathrm{mT}$ and $B_{\mathrm{z}}=1.2 \mathrm{mT}$, respectively. (C) Hahn echo free precession of the spin when prepared in the DPS at $\mathrm{B}=0$ $\mathrm{mT}$ (blue, $\left|\psi_{0}\right\rangle=\frac{1}{\sqrt{2}}(|+1\rangle+|-1\rangle)$ ), and outside of the DPS at B $=0 \mathrm{mT}\left(\right.$ red, $\left.\left|\psi_{0}\right\rangle=\frac{1}{\sqrt{2}}(|0\rangle+|+\rangle)\right)$ and at $B_{z}=1.2$ $\mathrm{mT}$ (orange, $\left.\left|\psi_{0}\right\rangle=\frac{1}{\sqrt{2}}\left(|0\rangle+\left|+1_{z}\right\rangle\right)\right)$. Error bars represent 1 SD. 

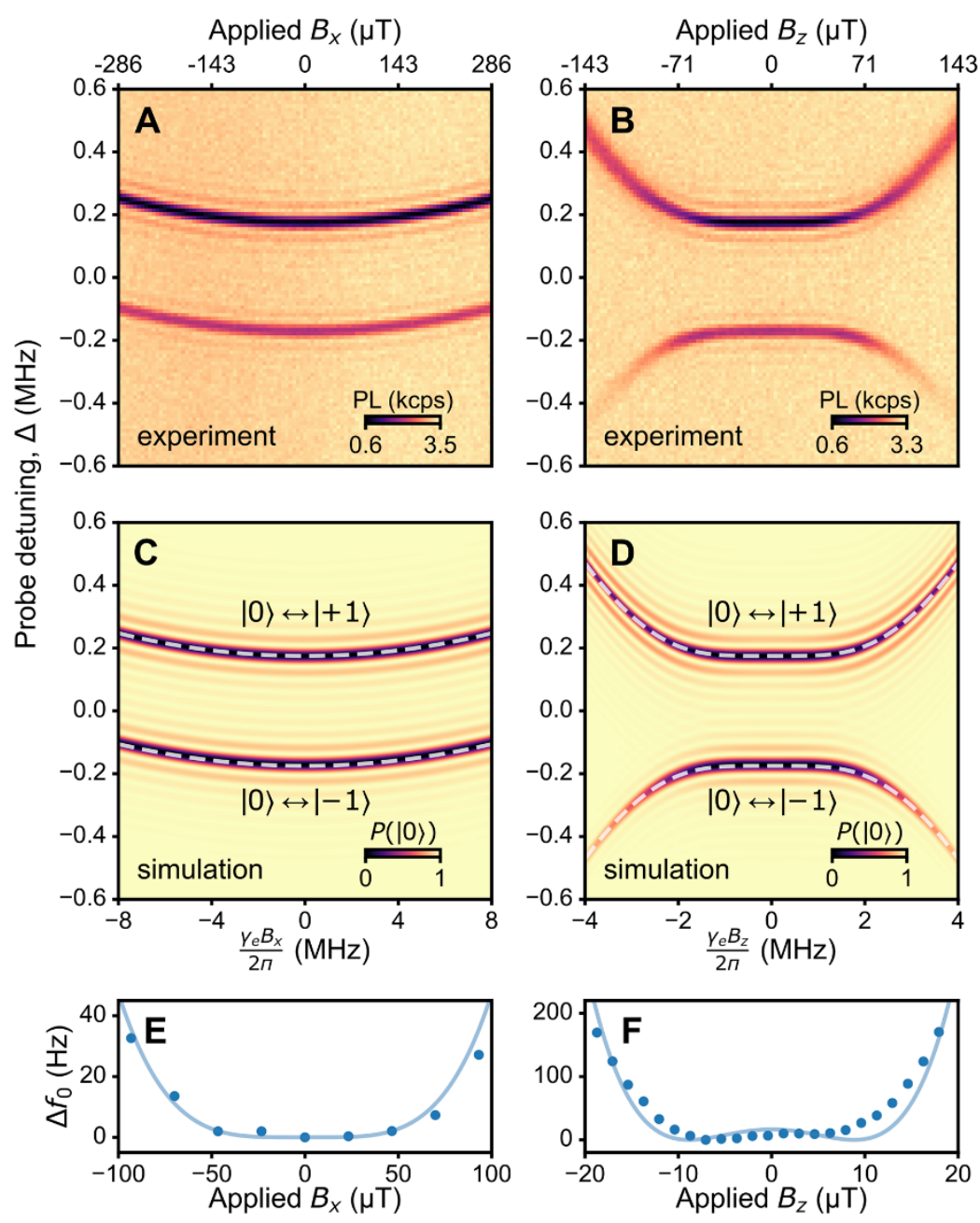

Fig. 3. Energy dispersion in the DPS. (A, B) Measured spectrum of the $|0\rangle \leftrightarrow| \pm 1\rangle$ transitions over a range of applied $x$-axis $(A)$ and $z$-axis (B) magnetic fields. The contrast recovery procedure (see Supplementary Materials) produces unequal photoluminescence contrast between the two resonances. The probe detuning, $\Delta$, is referenced to the $|0\rangle \leftrightarrow|+\rangle$ resonance frequency. In (B), high field induces inhomogeneous broadening, as the spin is no longer fully encoded in the DPS. (C, D) Simulated spin resonance spectrum of the driven $| \pm 1\rangle$ states corresponding to $(A)$ and $(B)$, respectively. Dashed white lines indicate transition energy spectra derived from Floquet analysis. ( $\mathrm{E}, \mathrm{F}$ ) Energy difference $\Delta f_{0}$ between the $|+1\rangle$ and $|-1\rangle$ states as a function of applied $B_{x}(E)$ and applied $B_{z}$ $(F)$, as measured using Ramsey interferometry of $\left|\psi_{0}\right\rangle=\frac{1}{\sqrt{2}}(|+1\rangle+|-1\rangle)$. Solid lines are the energy differences derived from Floquet analysis with no free parameters. Error bars are smaller than the symbol size. 


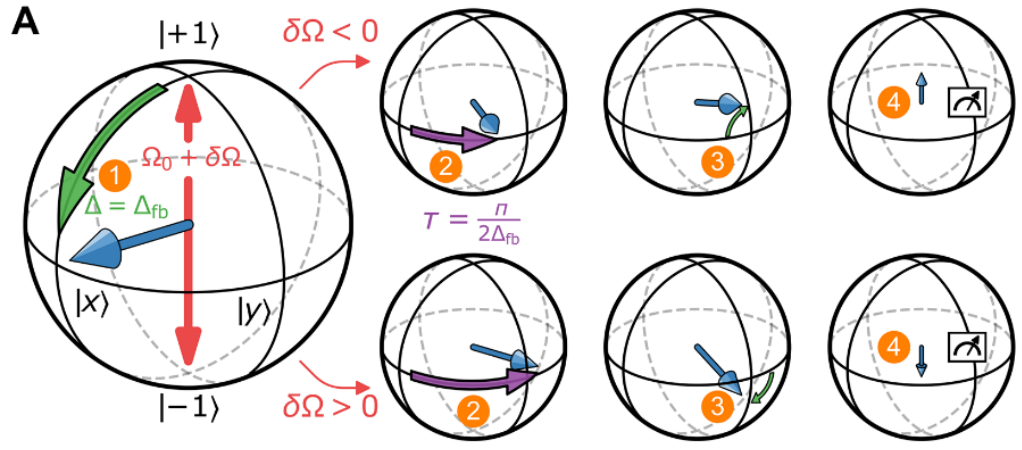

B

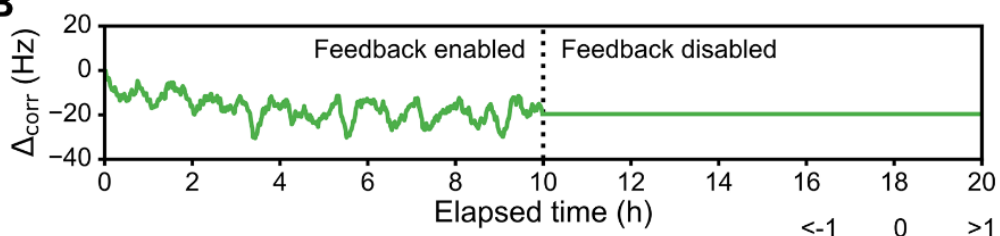

C

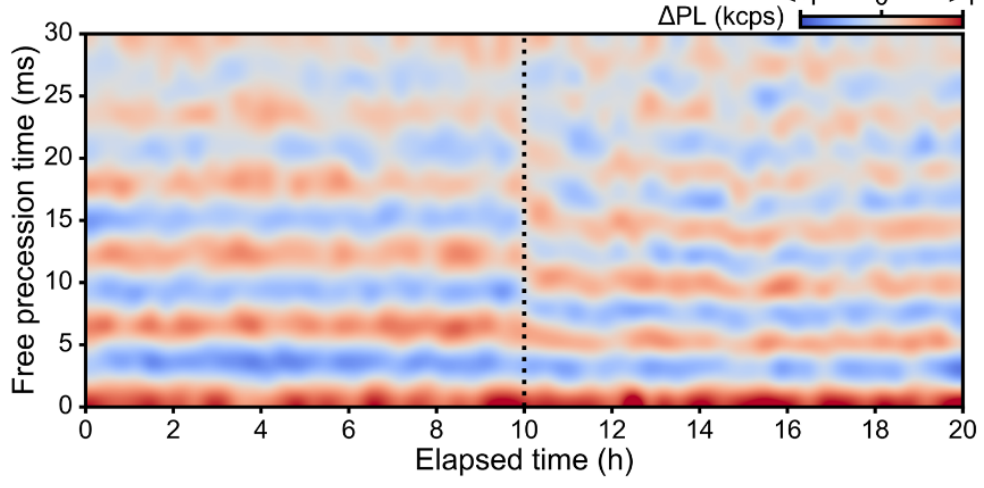

D

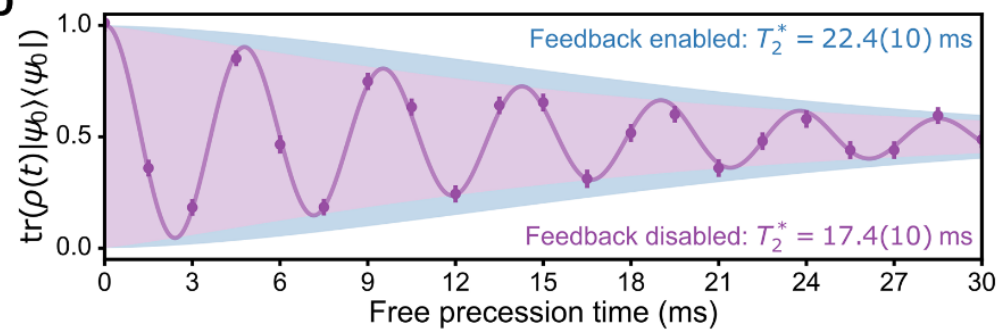

Fig. 4. Active spin resonance feedback. (A) Bloch spheres illustrating the spin resonance feedback protocol. See Methods for step-by-step details. (B) Applied corrective detuning to the frequency of the drive resonant with $|+1\rangle \leftrightarrow|-1\rangle$. During hours $0-10$, active feedback is applied to the spin and the corrective detuning is derived from a proportional factor of the measured error signal. (C) Stabilization of Ramsey free precession of $\left|\psi_{0}\right\rangle=\frac{1}{\sqrt{2}}(|+1\rangle+|-1\rangle)$ with active spin resonance feedback. While active feedback is enabled, the observed Ramsey fringes are stable at the chosen detuning of $+166.6 \mathrm{~Hz}$. Once disabled, slow drifts in the dressing drive Rabi frequency shift the effective detuning of the microwave pulse used to prepare the superposition. Interpolation applied to emphasize fringe locations. (D) The Ramsey free precession decay envelope when integrating the individual Ramsey free precession iterations in (C), demonstrating the increased inhomogeneity without the feedback process. Error bars represent 1SD. 